Zbigniew Waleszczuk

Catholic University of Eichstaett-Ingolstadt, Germany

\title{
Die Ökologie der Person - \\ Kantische Motive des Personbegriffs in Karol Wojtyłas "Person und Tat"
}

\section{The Ecology of Person \\ - Kantian Motives in the Concept of Person in Karol Wojtyła's "The Acting Person"}

\begin{abstract}
Apart from many legitimate concerns about ecology, the most important contemporary concern should be of anthropological nature and must address the human as a person (Benedikt XVI). What is man? Are human beings free? What should human beings do? These Kantian questions also describe the main starting points of Karol Wojtyła's philosophy in "The Acting Person". With regard to the importance of the question (ecology of man) our challenge is divided into three parts. Firstly, man is a person; secondly, his call is to liberty and lastly, responsible love is an emanation of personal dignity. In this manner the three important points in Wojtyła's philosophy (person, liberty, love) are well characterized. The essence of man is his personal dignity as a source for the possibility of free (responsible) decisions - "the act". The experience of morality is included in the experience of being a person. Wojtyła poses the question "how do I understand who I am throughout my acts?" Like Kant, the author emphasizes the importance of free will, conscientiousness as an obligation, which speaks to the conscious person. I determine myself through my own decision (person's actions). For Wojtyła, participation in love is the basis of all human personal experiences. "Only persons participate in love". Participation in the character of the other becomes - in the language of Wojtyła - "the choice of the other person in myself".
\end{abstract}


Der Begriff Ökologie ist heutzutage in aller Munde. Meistens jedoch wird er verstanden in Bezug auf die Natur (Verbrauch der Ressourcen, Ausbeutung der Natur, Bedrohung der Tierarten, Wasser- und Luftverschmutzung usw.), die quasi in Opposition zum Menschen präsentiert wird, so als ob der Mensch nicht ein Teil der Natur wäre. Das oben genannte Thema Ökologie der Person beabsichtigt den Anliegen des Papstes Benedikt XVI. entgegen zu kommen, der die bedrohende Tendenz feststellt, dass bei aller berechtigten Sorge um die Ökologie, die größte Sorge anthropologischer Natur ist und dem Menschen als Person gebührt ${ }^{1}$.

1869 hat der deutsche Biologe Ernst Haeckel als Erster den BegriffÖkologie als neue Wissenschaft definiert und beschreibt damit die Lehre über die Ökonomik der Natur, d.h. die Erforschung der Pflanzen und Tiere, mit ihrer eigenen organischen und anorganischenUmwelt, mit der sie direkt oder indirekt in Kontakt stehen. In den Quellen dieser Definition steht also ein Problem der Relationen, die verschiedene Ökosysteme verbinden. In den siebziger Jahren des vorigen Jahrhunderts wurde immer häufiger empfunden, dass die gegenwärtige Bewirtschaftungsweise und die Ausschöpfung der Ressourcen an ihre Grenzen stoßen. Der Mensch scheint sich seine eigenen Lebensgrundlagen zerstören zu wollen. Der 1968 gegründete „Club of Rome“ hat u.a. das Ziel der Bewusstseinsbildung der Weltbevölkerung über die Gefahren, die aus dem räuberischen Wirtschaftssystem entstanden sind und die als ökologische Katastrophe enden können². Im Jahre 1972 wurde „Die Grenzen des Wachstums“ eine bedeutende und für ökologische Initiativen wegweisende Schrift publiziert ${ }^{3}$. Leider wurde in der ökologischen Bewegung der Schwerpunkt auf die Erhaltung der Pflanzen- und Tierwelt gelegt. Dabei führten die Säkularisierung und die Reduzierung der Perspektiven auf das Diesseits dazu, dass man die „Spuren Gottes“ in der Welt ignorierte ${ }^{4}$. Es ist deshalb, aus

\footnotetext{
${ }^{1}$ Benedikt XVI., Caritas in Veritate, Vatican 2009.

2 Beispielsweise sagten die katholischen Bischöfe auf der Bischofssynode „Über die Gerechtigkeit in der Welt" schon 1971, noch vor der Stockholmer 6. Umweltkonferenz und dem ersten Bericht des Club of Rome: „Unerfindlich ist, wie die reichen Völker es rechtfertigen wollen, ihren Zugriff auf die Güter der Erde noch weiter zu steigern, wenn das zur Folge hat, dass entweder die anderen Völker niemals über ihre elende Notlage hinauskommen oder gar die physischen Grundlagen des Lebens auf der Erde Gefahr laufen, zerstört zu werden“. (Iustitia in Mundo 64-7). Zit. Nach: G. Kruip, http://www.sozialethik.kath theologie.uni-mainz.de/antritt. doc.pdf (30.08.2012).

${ }^{3}$ D. Meadows, Die Grenzen des Wachstums, Stuttgart 1972; The Limits to Growth. Universe Books, 1972.

4 Die kulturelle Krise hat ihre philosophischen Wurzeln in der neuzeitlichen anthropologischen Wende, die den Menschen nach dem Prinzip „etsi Deus non daretur” zum ausschließlichen Schöpfer seiner selbst macht.
} 
der Sicht der Kirche, ein wichtiges Zeichen der Zeit ${ }^{5}$, die Personenwürde des Menschen zu verteidigen gegenüber allen reduzierenden, positivistischen und materialistischen Menschenbildern ${ }^{6}$. Robert Spaemann erinnert daran, dass seit Immanuel Kant der Personbegriff zum zentralen Begriff bei der Begründung von Menschenrechten wurde? ${ }^{7}$ Leider ist es in der letzten Zeit, wie Spaemann feststellt, zu dramatischen Veränderungen in unserer Kultur gekommen. „Der Personbegriff spielt plötzlich eine Schlüsselrolle bei der Destruktion des Gedankens, Menschen hätten, weil sie Menschen sind, gegenüber ihresgleichen so etwas wie Rechte. Nicht als Menschen sollen Menschen Rechte haben sondern nur, soweit sie Personen sind. Nicht alle Menschen aber und nicht Menschen in jedem Stadium ihres Lebens und in jeder Verfassung ihres Bewusstseins sind, so wird uns gesagt, Personen“"8.

In seiner Ansprache im deutschen Bundestag beschreibt Papst Benedikt XVI. die Situation der gegenwärtigen Menschen als eine ökologische Frage, die unsere Existenz entscheidend prägt ${ }^{9}$. Der Papst will uns daran erinnern, dass die Grundlage jeder Rechtsbildung die Fähigkeit ist das Gute vom Bösen unterscheiden zu können. Benedikt erinnert in seiner durch Säkularisierung und einseitige (links-liberale) Ökologie beeinflussten Heimat daran, dass

${ }^{5}$ Der Terminus Zeichen der Zeit, stammt aus der Enzyklika „Pacem in Terris“ Johannes XXIII., der die Aufgabe der christlichen Sozialethik anknüpfend an die Forderung Jesu, die Zeichen der Zeit zu erkennen, als die Hauptaufgabe und als Einladung zu verantwortungsbewusstem Handeln an jeden Christen richtet. W. Kirchschläger schreibt dazu: „Johannes XXIII. hat der Kirche damit das Grundmodell und die Methode für ihr eigenes Leben in Erinnerung gerufen und zugleich angedeutet, wie dies zu verwirklichen ist: Nicht, indem auf die Unglückspropheten gehört wird, die aus einer nach rückwärtsgewandten Perspektive die Gegenwart schlecht machen, sondern indem im Vertrauen auf das Wirken des Geistes die Kirche sich anschickt, „einen Sprung nach vorwärts“" zu wagen, um ,in der Welt von heute“ die christliche Botschaft zu verkündigen“. http:// www.unilu.ch/files/die-zeichen-der-zeit-erkennen.pdf (30.08.2012).

${ }^{6}$ Siehe: W. Schweidler, Über Menschenwürde. Der Ursprung der Person und die Kultur des Lebens, Wiesbaden 2012. W. Kasper, Barmherzigkeit. Grundbegriff des Evangeliums - Schlüssel christlichen Lebens, Herder 2012, S. 14-15. „(...)the concept of a person can be applied to some higher animals. Some philosophers hold that apes and dolphins should be recognized as persons". G. Hołub, Being a Person and acting as a Person, Forum Philosophicum 13 (2008), S. 269.

${ }^{7}$ R. Speamann, Personen, Versuche über den Unterschied zwischen ,etwas “ und ,jemand“. Klett-Cotta, Stuttgart 1996, S. 10.

${ }^{8}$ Ebenda. „Human embryos or even fetuses cannot be called rational, intelligent and selfconscious entities, at least in an active sense. Because of the limited development of the cerebralnervous system, they cannot perform such activities, and in this self-deployed manner of thinking they cannot be counted as persons". G. Hołub, Being a Person and acting as a Person, Forum Philosophicum 13 (2008), S. 270.

${ }^{9}$ Benedikt XVI., In Gott ist unsere Zukunft, Leipzig 2011. Benedikt XVI., Wir müssen anders leben! Damit die Schöpfung überleben kann, Herder 2012; C. Breuer, Die Ökologie des Menschen, in: J. Kreiml, Neue Ansage des Glaubens, Regensburg 2012, S. 114-149. 
die Gestalt des jungen Herrschers Salomo und seine Bitte an Gott um die Weisheit - jene Unterscheidungsgabe, die nicht nur für die Parlamente in ihrem Bemühen das Recht zu formulieren, sondern uns allen als moralischen Wesen eine Lehre erteilt ${ }^{10}$. Ohne Hinwendung zu Gott beraubt sich der Mensch seiner eigenen Verantwortlichkeit ${ }^{11}$, denn ohne Transzendenz fallen die Werte leicht dem Positivismus und Relativismus der jeweiligen Mehrheiten zum Opfer. Man braucht heute, mehr denn je, dringend eine Ökologie der Person ${ }^{12}$, die die Würde des Menschen ohne Ausnahme als unantastbar nachhaltig definiert und verteidigt ${ }^{13}$. Solche Ökologie können wir (laut Papst Benedikt) nicht ohne Gott ${ }^{14}$ menschenwürdig formulieren und gestalten. Auch der Vorgänger Benedikts, Papst Johannes Paul II. sprach im gleichen Sinne. Bevor wir über die Pflanzen

${ }^{10}$ „Ich denke, auch heute können wir letztlich nichts anderes wünschen als ein hörendes Herz - die Fähigkeit, Gut und Böse zu unterscheiden (...)“. Benedikt XVI., In Gott ist unsere Zukunft, S. 39.

${ }^{11}$ „Im Sinne Kants kann man von einem Postulat sprechen, das besagt: wenn absolute Würde des Menschen sein soll, dann nur, wenn Gott ist und wenn ein Gott des Erbarmens und der Gnade ist“. W. Kasper, Barmherzigkeit..., S. 14.

${ }^{12}$ „Naturalists contend, therefore, that the person is not only a temporal phenomenon but also a kind of exclusive feature of a given being, e. g. a human being. It is a sort of 'label' which is attached to a human being in moments when he/she is physically and mentally unimpaired and fully flourishing. It follows, according to this view, that in some moments of life, we humans are persons, and in others we are not". G. Hołub, Being a Person and acting as a Person, Forum Philosophicum 13 (2008), S. 273.

13 „Sind alle Menschen Personen? Es zeigt sich, daß die bejahende Antwort Voraussetzungen hat. Sie setzt voraus, daß Personen zwar a priori in einer auf Anerkennung basierenden wechselseitigen Beziehung stehen, aber daß diese Anerkennung nicht dem Personsein als dessen Bedingung vorausgeht, sondern auf einen Anspruch antwortet, der von jemandem ausgeht. Sie setzt ferner voraus, daß wir diesen Anspruch zwar aufgrund gewisser Artmerkmale zuerkennen, daß es aber für die Anerkennung als Person nicht auf das tatsächliche Vorhandensein dieser Merkmale ankommt, sondern nur auf die Zugehörigkeit zu einer Art, deren typische Exemplare über diese Merkmale verfügen. Und dies, obwohl oder weil Personen sich zu der Art, der sie angehören, auf andere Weise verhalten als Exemplare anderer Spezies. Personen sind in einem unvergleichlichen Sinn Individuen. Gerade deshalb kommt es für ihre Anerkennung als Personen nicht auf das individuelle Auftreten bestimmter Artmerkmale an, sondern nur auf die Zugehörigkeit zur Art“. R. Spaemann, Personen, Versuche über den Unterschied zwischen „etwas" und ,jemand“. Klett-Cotta, Stuttgart 1996, S. 11.

${ }^{14}$ „Kant hat freilich auch erkannt, dass es seinen Preis haben würde, angesichts des Übels in der Welt den Gottesgedanken aufzugeben. Denn soll die Würde des Menschen trotz der Erfahrung des Bösen Bestand haben, so ist das nur dann möglich, wenn man am Gottesgedanken als einem Postulat der praktischen Vernunft festhält. Nur unter der Voraussetzung Gottes, der menschliche Freiheit und die Naturordnung umgreift, ist Hoffnung auf eine Versöhnung von menschlicher Freiheit und Naturschicksal möglich. Der Gottesgedanke verwahrt in sich die Hoffnung auf das Gelingen menschlicher Freiheit. Diese Hoffnung aufgeben hieße den Menschen aufgeben (...)“. W. Kasper, Barmherzigkeit..., S. 14. 
und Tiere sprechen sollen wir zuerst die Unantastbarkeit der Person und ihrer Würde definieren ${ }^{15}$.

Der vorliegende Beitrag beabsichtigt deshalb nicht über ökologische Fragen in klassischem Sinne zu referieren, sondern ist anthropologisch ausgerichtet: Was ist der Mensch? Ist der Mensch frei? Was soll er tun? Diese kantischen Fragen umschreiben auch gut die drei wichtigsten Ansatzpunkte der Philosophie Karol Wojtylas in seinem Hauptwerk „Person und Tat“, das 1969 erstmals in polnischer und 1981 in deutscher Sprache erschien ${ }^{16}$. Im Hinblick auf die Wichtigkeit der Frage (Ökologie des Menschen) gestaltet sich unsere Aufgabe folgendermaßen: 1. Der Mensch ist eine Person; 2. Zur Freiheit berufen; 3. Verantwortliche Liebe als Emanation personaler Würde.

\section{Der Mensch ist eine Person}

Wojtyla betont in seinem philosophischen Denken, dass die Beobachtung der Taten eine spezifisch menschliche Eigenschaft darstellt ${ }^{17}$. Gerade durch die menschliche Praxis können wir laut dem Philosophen aus Krakau, die Verwirklichung des Menschseins als Person erkennen. In den menschlichen Handlungen - in der Tat - manifestiert sich die Person. Der Mensch hat Anteil

${ }^{15}$ W. Schweidler, Das Unantastbare: Beiträge zur Philosophie der Menschenrechte, Lit. Verlag 2001. Ders. Über Menschenwürde. Der Ursprung der Person und die Kultur des Lebens, Wiesbaden 2012.

${ }^{16}$ K. Wojtyła, Person und Tat, Herder 1981; Ders., Osoba $i$ czyn oraz inne studia antropologiczne, Towarzystwo Naukowe KUL, Lublin 2000.

${ }^{17}$ „Stajemy [...] na stanowisku - powiada autor Osoby $i$ czynu - że czyn jest szczególnym momentem danym w oglądzie - czyli doświadczeniu - osoby", K. Wojtyła, Osoba i czyn oraz inne studia antropologiczne, Towarzystwo Naukowe KUL, Lublin 2000, S. 58, 59. „Czyn nie jest bowiem czymś w ścisłym sensie odrębnym od osoby: czyn to osoba w jej akcie wyrażania i konstytuowania samej siebie. Osoby są ponadto bytami społecznymi i ukierunkowanymi na życie we wspólnocie. Realizują się jako osoby poprzez interakcje z innymi podmiotami, poprzez ofiarowywanie i przyjmowanie. Aby ukazać, jak pogodzić dobro wspólnoty z dobrem jej indywidualnych członków, Wojtyła zaproponował teorię uczestnictwa. Uważał, że wszyscy ludzie powinni wnosić wkład do dobra wspólnego, które ma następnie służyć pożytkowi poszczególnych jednostek. To nauczanie na temat uczestnictwa i dobra wspólnego zawiera w sobie ukrytą krytykę nie tylko marksistowskiego kolektywizmu, ale również libertariańskiego indywidualizmu i anarchistycznej alienacji”. A. Dulles, Jan Pawet II wobes tajemnicy osoby ludzkiej, „Ethos” 19 (2006) nr 4 (76), s. 39-53. „He does not want the person to be presupposed. Rather, action reveals the person, and we look at the person through his action. . . Action gives us the best insight into the inherent essence of the person and allows us to understand the person most fully. K. Wojtyła, Acting Person, Springer 1979, S. 11; A. Ong, John Paul It's Philosophy of the Acting Person: A Personalistic Approach to Life, Edwin Mellen Press Ltd, 2008, S. 78. 
an der Wahrheit, erkennt die Wahrheit durch seine Praxis. Die anthropologische Frage, die schon Immanuel Kant als zentrale Frage ${ }^{18}$ der Philosophie beschreibt: „Wer ist der Mensch?“ ist die Hauptfrage aller philosophischen Untersuchungen auch für Karol Wojtyla ${ }^{19}$. Als Antwort auf die oben gestellte Frage formuliert er anknüpfend an Kant: der Mensch ist eine Person ${ }^{20}$. Seine Würde ist einzigartig und einmalig und unveräußerlich, unabhängig von den Umständen seiner Existenz $^{21}$. Was im Menschen unreduzierbar ist, ist die Perspektive, die ihn gleichzeitig als Subjekt und Objekt der Erfahrung begleitet (Selbstbewusstsein) ${ }^{22}$. Wojtyla zeigt, dass der Mensch sich selbst erkennt als Subjekt, indem er die objektive Wahrheit erkennt. „Die Erfahrung jedes sich außerhalb des Menschen befindlichen Gegenstandes ist immer mit einer Erfahrung des Menschen selbst verbunden. Der Mensch erfährt nie etwas außerhalb seiner selbst ohne in dieser Erfahrung auch irgendwie sich selbst zu erfahren“23. Selbstverständlich geht es ihm nicht darum in Subjektivismus zu verfallen, es scheint jedoch, dass in

${ }^{18}$ Kant betont, dass der Mensch unter zwei Blickwinkeln (Leib und Seele) zu betrachten ist, sie folgen aus der Beantwortung der drei Fragen: „1. Was kann ich wissen? 2. Was soll ich tun? 3. Was darf ich hoffen?“. I. Kant, Kritik der reinen Vernunft, Köln 2011, B832f. Als vierte Frage fügt er bei: „Was ist der Mensch?“. Dazu sagt er: „Die erste Frage beantwortet die Metaphysik, die zweite die Moral, die dritte die Religion, und die vierte die Anthropologie“. N. Fischer schreibt dazu: Kant denkt den Menschen als Wesen der Metaphysik, der Moral und die Religion (Logik A 25) und kann damit als ein Philosoph gelten, der sich in hervorragender Weise um die philosophischen Grundfragen der (christlichen) Theologie bemüht hat (wovon er auch selbst gesprochen hat)“. N. Fischer, Sein und Sinn der menschlichen Freiheit in der Philosophie Kants, in: Ch. Böttigheimer, N. Fischer, M. Gerwing (Hg.), Sein und Sollen des Menschen, Münster 2009, S. 26.

${ }^{19}$ K. Wojtyła, A. Szostek, T. Styczeń, Der Streit um den Menschen. Personaler Anspruch des Sittlichen, Kevelaer 1979.

${ }^{20}$ J. Galarowicz, Człowiek jest osoba, Krakow 1994; J. Schwartländer, Der Mensch ist Person, Mainz 1968.

${ }^{21}$ „Ein jeder Mensch“, schreibt Kant in der Metaphysik der Sitten, 2. Teil, § 38, ,hat rechtmäßigen Anspruch auf Achtung von seinen Mitmenschen, und wechselseitig ist er dazu auch gegen jeden anderen verbunden“. I. Kant, Metaphysik der Sitten, 2. Teil, A 139, in: Weischedel W. (Hg.), Immanuel Kant. Werke in sechs Bänden, Wissenschaftliche Buchgesellschaft, Darmstadt (1956-1964), Bd. 4, S. 600. „Achtung geht jederzeit auf Personen, niemals auf Sachen“, I. Kant, Kritik der praktischen Vernunft A, S. 35. Siehe: N. Fischer, Sein und Sinn der menschlichen Freiheit, S. 31.

22 “(...) świadomości zawdzięcza człowiek upodmiotowienie tego co przedmiotowe”, K. Wojtyła, Osoba i czyn oraz inne studia antropologiczne, Towarzystwo Naukowe KUL, Lublin 2000, S. 91. Vgl. Hanns-Gregor Nissing (Hg.), Einleitung und Übersetzung, in: K. Wojtyla, Wer ist der Mensch? Skizzen zur Anthropologie, München 2011, S. I-XVI. Siehe: K. Wojtyła, Subjectivity and Irreducible in Man, "Analecta Husserliana", Vol. VII, 1978. (Subjektivität und das Unreduzierbare" im Menschen), in: Nissing (Hg.), Wer ist der Mensch?, S. 3-12.

${ }^{23}$ K. Wojtyła, Osoba i czyn..., S. 67; „Ich vollziehe nicht nur äußerlich eine gute oder schlechte Tat, sondern werde dadurch innerlich gut oder schlecht“. Vgl. K. Wojtyła, Osoba i czyn..., S. 204. 
diesem Punkt zum besseren Verständnis einige Voraussetzungen ausführlicher behandelt werden könnten. Ähnlich wie später Robert Spaemann betont er, dass der Mensch „Jemand“ und nicht „Etwas“ ist ${ }^{24}$. Ich erlebe mich, laut Wojtyla in „Person und Tat“, als ein konkreter Seiender und nicht nur als ein nur im Bewusstsein konstituierendes Phänomen. Im Gegensatz zum Subjektivismus, den er für einen theoretischen Irrtum hält, unterstreicht er den Realismus in seiner Anthropologie ${ }^{25}$. Den Begriff der Autoteleologie ${ }^{26}$ hat der Autor auch von Kant übernommen. Seine Formulierung des kategorischen Imperativs zeigt die Person als Zweck an sich selbst, die durch die ihr innewohnende Freiheit zu verantwortlichen Handlungen gemäß den moralischen Gesetzen bestimmt ist ${ }^{27}$. Kant erfasst auch „die moralischen Gesetze zugleich als Gebote Gottes. Er sagt (KpV A 233): »Auf solche Weise führt das moralische Gesetz durch den Begriff des höchsten Guts, als das Object und den Endzweck der reinen praktischen Vernunft, zur Religion, d.i. zur Erkenntniß aller Pflichten als göttlicher Gebote, nicht als Sanctionen, d.i. willkürliche, für sich selbst zufällige Verordnungen eines fremden Willens, sondern als wesentlicher Gesetze eines jeden freien Willens für sich selbst, die aber dennoch als Gebote des höchsten Wesens angesehen werden müssen «" $" 28$. Das Wesen des Menschen ist seine personelle Würde als Quelle der Möglichkeit zu freien (verantwortlichen) Entscheidungen - „der Tat““. Die Erfahrung der Moral ist in der Erfahrung der Person enthalten. Wojtyla verweist auf das personale „esse“ als Quelle der Tat und gleichzeitig als das „fieri“ der Person (Autokreation durch die Taten). Der Mensch als Person

${ }^{24}$ R. Spaemann, Personen. Versuche über den Unterschied zwischen ,etwas" und ,jemand“. Klett-Cotta, Stuttgart 1996.

${ }^{25}$ Vgl. J. Kupczak, Destined for Liberty. The Human Person in the Philosophy of Karol Wojtyła/ John Paul II., Washington D.C. 2000.

${ }^{26}$ „Das menschliche Handeln, die Tat, wendet sich unterschiedlichen Zielen, verschiedenen Objekten und Werten zu. Doch indem sich der Mensch auf diese verschiedenen Ziele, Objekte und Werte ausrichtet, wird im bewußten Handeln unumgänglich auch er selbst sich zum Ziel. Er kann nicht andere Objekte und Werte wählen, ohne dadurch zugleich über sich selbst und seinen eigenen Wert zu bestimmen. Damit wird er tatsächlich für sich selbst als Subjekt das erste Objekt. Die Struktur der menschlichen Tat ist in besonderem Maße autoteleologisch“. In: K. Wojtyła, A. Szostek, T. Styczeń, Der Streit um den Menschen. Personaler Anspruch des Sittlichen, Kevelaer 1979, S. 28.

${ }^{27}$ „Wenn in deinem Verhalten eine Person Objekt deines Handelns ist, vergiss niemals, dass du sie nicht bloß als Mittel, als Werkzeug behandeln darfst, sondern sei dir bewusst, dass sie ihr eigenes Ziel hat oder wenigstens haben sollte“. K. Wojtyła, Liebe und Verantwortung, S. 25.

${ }^{28}$ N. Fischer, Müssen Katholiken weiterhin Furcht vor Kant haben? Kants Philosophie als sancilla theologiae`, Katholische Universtät Eichstätt-Ingolstadt, Kastner 2005, S. 12. 
gestaltet nicht nur seine äußere Welt, er schafft auch sich selbst ${ }^{29}$. Die Person ist als Seiendes das Vollkommenste aller Seienden (Thomas von Aquin) ${ }^{30}$.

Die Person ist aufgrund ihrer Transzendenz ${ }^{31}$ auf die anderen Personen ausgerichtet. Der Mensch, als „ens sociale“ braucht zum Leben verschiedene Gemeinschaften. In diesem Sinne steht meine Tat in Zusammenhang mit meiner Selbstverwirklichung. „Ich vollziehe nicht nur äußerlich eine gute oder schlechte Tat, sondern werde dadurch innerlich gut oder schlecht ${ }^{\star “ 32}$. Die klassische thomistische Philosophie hat der Problematik ,actus humanus“ viel Platz eingeräumt. Diese Interpretation jedoch unterscheidet nicht zwischen meinen eigenen Handlungen- ,ich handle“ und meinen passiven Erfahrungen - „etwas geschieht mit mir“. Die Quelle des Wissens ist für Wojtyla die Erfahrung des Menschen ${ }^{33}$. Für den Philosophen aus Krakau offenbart die innere Erfahrung zwei Typen der Aktivität des Menschen: - etwas geschieht in mir

${ }^{29}$ Fakt „człowiek działa” zawiera w sobie nie tylko spełnienie czynu na zasadzie samostanowienia, ale zawiera również spełnienie osoby (...). K. Wojtyła, Osoba i czyn, S. 219.

30 ,aliquid perfectissimum in tota natura“.http://www.corpusthomisticum.org/sth1028. html. „S. Tommaso ha un concetto altissimo della persona. Questa a suo giudizio è quanto di più perfetto esiste nell'universo: "Persona significat id quod est perfectissimum in tota natura, scilicet substantia in natura rationalis" (I, q. 28, a. 3). Egli guarda alla persona dal punto di vista ontologico e la considera quindi come una modalità dell'essere, ossia di quella perfezione che nella sua filosofia è la perfectio omnium perfectionum e l'actualitas omnium actuum, ed è proprio rispetto a questa perfezione che la persona occupa il gradino più alto: l'essere nella persona trova la sua attuazione più piena, più eccellente, più completa. Per questo motivo tutti gli enti che si fregiano del titolo di persona, sono enti che godono di una dignità infinita, di un valore assoluto: sia che si tratti di Dio, degli angeli o dell'uomo. Il concetto di persona è un concetto analogico: non si predica allo stesso modo, ossia univocamente, di Dio, degli angeli e dell'uomo, ma secondo un ordine di priorità e posteriorità (secundum prius et posterius); tuttavia, esso designa sempre la stessa perfezione fondamentale: il sussistere individuale nell'ordine dello spirito. Come dice $\mathrm{S}$. Tommaso col suo linguaggio sobrio e preciso: "Omne subsistens in natura rationali vel intellectuali est persona" (C. G., IV, c. 35)". http://digilander.libero.it/avemaria78/ tommaso/termini/persona.htm (30.08.2012). B. Davies, The Thought of Thomas Aquinas, Oxford 1992; G.K. Chesterton, Der Heilige mit dem gesunden Menschenverstand, Herder 1957.

${ }^{31}$ „Transzendenz ist im modernen Denken so etwas wie ein anderer Name für Person“. K. Wojtyla, Die Person: Subjekt und Gemeinschaft, in Nissing (Hg.), Wer ist der Mensch?, Skizzen zur Anthropologie, München 2011, S. 126.

${ }^{32}$ Ebenda, S. XLI.

33 „Nach Kant ist unser menschliches Erkenntnisvermögen begrenzt; metaphysische Spekulationen über Fragen, welche den Bereich menschlicher Erfahrung übersteigen, sind uns grundsätzlich nicht möglich“. „Denn in der Tat scheitern alle Versuche der Theodizee sowohl an der Ehrfurcht vor Gott und am unergründlichen Geheimnis seines Willens wie an der Ehrfurcht vor dem Geheimnis des Menschen und seines Leidens. Erst recht lässt sich das Verhältnis von Gott und Welt, von göttlicher und menschlicher Freiheit nicht von einem höheren Standpunkt aus in einen beide umgreifenden, sei es weisheitlichen oder dialektischen Sinn- und Ordnungszusammenhang einfügen. Bei jedem solchen Versuch erheben wir uns über Gott und 
oder mit mir (passives Erlebnis), - Ich als Subjekt handle (aktive Tat). Das erste beschreibt er mit dem Begriff „patio“, und er unterscheidet dies von „agere“ der selbstbestimmenden Entscheidung - actus hominis ${ }^{34}$. Wojtyla versucht damit aus der Grundlage des Thomismus (Realismus und Objektivität) eine Ethik zu entwerfen, die auch kantische Motive einbezieht ${ }^{35}$. Besonders deutlich zeigt sich das in der Frage, wie eine Ethik zu entwerfen sei. Im klassischen Eudaimonismus (Thomas von Aquin) bildet die Idee eines letzten Zieles und die Frage nach dem Glück den Maßstab des menschlichen Handels (bonum est quod omnia appetunt $)^{36}$. Anstatt thomistisch über das letzte Ziel zu sprechen, beginnt Wojtyla seine Untersuchungen in „Person und Tat" mit der unmittelbaren „Erfahrung des sittlichen Sollens“37. Nach Wojtyla sei „nicht die Frage nach Glück Prinzip und Maßstab des ethischen Sollens, sondern umgekehrt bedinge die Erfüllung des sittlichen Sollens die Glückserfahrung“383.

\section{Zur Freiheit berufen}

Das ganze Denken Wojtylas können wir auf einen Punkt bringen, der sich in der Frage formulieren lässt: Ist der Mensch frei? Die Urheberschaft, Selbstbestimmung ${ }^{39}$, Transzendenz, Gewissen, moralische Handlungen des Menschen - all das hängt zusammen mit der Antwort auf die oben gestellte Frage. Die Schlüsselbedeutung der Freiheit ${ }^{40}$ zeigt sich in ,jene(m) Moment, das das Handeln einer Person eindeutig von allem unterscheidet, was in ihr

sein Tun und versuchen, ihn von einer angeblich höheren Warte aus zu beurteilen, was Arroganz und Hybris ist". W. Kasper, Barmherzigkeit..., S. 126.

${ }^{34}$ Diese Differenzierung geht psychologisch tiefer in der Analyse der menschlichen Handlung als dies die klassische thomistische Philosophie getan hat.

${ }^{35}$ H-G. Nissing (Hg.), Wer ist der Mensch?, Einleitung, München 2011, S. XVII-XVIII.

${ }^{36}$ Vgl. Aristoteles, Nikomachische Ethik I 1 (1094a1). Vgl. H.G. Nissing (Hg.), Wer ist der Mensch?, Einleitung, München 2011, S. XVIII.

${ }^{37}$ „Die Unbedingtheit der sittlichen Erfahrung und deren Sollensanspruchs sowie von hier aus die Bedeutung des Gewissens wiesen ihn in die Richtung der Ethik Kants“. Ebenda.

${ }^{38}$ Ebenda.

39 „Interpretatorzy myśli Wojtyły przywiązują na ogół zbyt małą wagę do wpływu Kanta na uformowanie się jego antropologii filozoficznej, w szczególności jego teorii samostanowienia. Czynią tak - moim zdaniem - niesłusznie". J. Galarowicz, Człowiek jest osoba, Kraków 1994, S. 177.

${ }^{40}$ G. Weigel, Der Papst der Freiheit, Paderborn 2011. 
lediglich geschieht ${ }^{\star 41}$. Der Begriff Person beschreibt das Faktum: der Mensch handelt. Wojtyla stellt die Frage: Wie lässt sich aus meinen Taten verstehen wer ich bin? Deshalb beginnt er seine Untersuchungen mit der Problematik des Bewusstseins. Er stellt die Frage: was heißt es, mich selber als Autor meiner Taten zu verstehen? Um die Taten zu vollbringen, nimmt der Mensch die Wahrheit über sich selbst und über die Welt wahr (er transzendiert sich selbst). Diese Erfahrung erlaubt ihm die Entscheidungen zu treffen. Die wahre Freiheit ist erst dann möglich, wenn der Mensch zwischen Gut und Böse unterscheiden kann. Ohne Überprüfung der Relation von Wille zu Wahrheit, die gleichzeitig als ein wahres Gut empfunden und verstanden wird, kann keine Entscheidung vollbracht werden. Diese Abhängigkeit von der Wahrheit ${ }^{42}$ berührt die Grenzen der menschlichen Autonomie. Erst durch die Anerkennung der Wahrheit kann der Mensch handeln. In der Erfahrung der Schuld, des moralisch Bösen, sowie auch im Gegenteil dem Guten und Liebenden sieht Wojtyla - ähnlich wie Kant - die allen menschlichen Personen gebührende Würde ${ }^{43}$, die aus der inneren Freiheit

${ }^{41}$ Kants Lehre von der Personenwürde des Menschen können wir folgendermaßen beschreiben: Was der Mensch in der Sinnenwelt will, das sei keine Freiheit, sondern gesteuert über Bedürfnisse, über Triebe und Zwecke. Hier herrsche keine Freiheit, weil alle Handlung in den Ketten der Naturnotwendigkeit liege. Freiheit gebe es erst dort, wo sich der Mensch von seinen sinnlichen Wünschen und Trieben löse und ins Reich der Vernunft wechsle. Dort erkennt er das moralische Gesetz - darin liege seine Würde als Mensch - im kategorischen Imperativ. Siehe z.B.: A. Winter, Der andere Kant. Zur philosophischen Theologie Immanuel Kants, HildesheimZürich-New York 2000.

${ }^{42}$ Die thomistische Reflexion bleibt deshalb für die Philosophie Wojtylas so relevant, weil sie die ganze Struktur des Kosmos, der Welt und des Menschen auf Gott hin orientiert. Gleichzeitig erkennt Wojtyla, dass der Thomismus allein nicht ausreicht, um der Dynamik der anthropologischen Frage gerecht zu werden. Bei Kant lesen wir folgendes Bekenntnis: „(..) die wichtigste aller unserer Erkenntnisse: Es ist ein Gott" (Kants Werke, Akademieausgabe 2,65 [Fettdruck im Original] ); „Diese unvermeidlichen Aufgaben der Vernunft selbst sind Gott, Freiheit und Unsterblichkeit. Die Wissenschaft aber, deren Endabsicht mit allen ihren Zurüstungen eigentlich nur auf die Auflösung derselben gerichtet ist, heißt Metaphysik“" (3,31); „Die Metaphysik hat zum eigentlichen Zwecke ihrer Nachforschung nur drei Ideen: Gott, Freiheit und Unsterblichkeit, ... Alles, womit sich diese Wissenschaft sonst beschäftigt, dient ihr bloß zum Mittel, um zu diesen und ihrer Realität zu gelangen“. Zit. nach. A. Winter, Der andere Kant. Zur philosophischen Theologie Immanuel Kants, Hildesheim-Zürich-New York 2000, S. XIV.

${ }^{43}$ Kant schreibt: „Ein jeder Mensch hat rechtmäßigen Anspruch auf Achtung von seinen Nebenmenschen, und wechselseitig ist er dazu auch gegen jeden anderen verbunden." Und fährt fort: „Die Menschheit selbst ist eine Würde; denn der Mensch kann von keinem Menschen (weder von Anderen noch sogar von sich selbst) blos als Mittel sondern muß jederzeit zugleich als Zweck gebraucht werden, und darin besteht eben seine Würde (die Persönlichkeit), dadurch er sich über alle anderen Weltwesen, die nicht Menschen sind und doch gebraucht werden können, mithin über alle Sachen erhebt.“ I. Kant, Metaphysik der Sitten, Frankfurt am Main 2007, S. 462 Z. 18ff. Vgl. http://www.rechtsphilosophie.uni-goettingen.de/ZurWuerdeDesMenschenBeiKant.pdf (30.08.2012). 
hervorgeht. Für Wojtyla ist diese Frage fundamental. Erst durch die Erkenntnis der Wahrheit kann das Subjekt das Gute wählen und handeln. Einen besonderen Schwerpunkt seiner Ausführungen legt der Kardinal von Krakau deshalb auf das Gewissen. Die Rolle des Gewissens beschreibt er als „Stimme Gottes“, oder Kant folgend - als Gesetzgeber der Person ${ }^{44}$. Die Tat ist immer eine Synthese der Dynamik des Menschen, neben meinem Willen werden auch psychophysische Aspekte integriert. Im Gegensatz zu den unbeweglichen und höchst abstrakten Konstruktionen der klassischen Philosophie, bemüht sich Wojtyla den ganzen Menschen im Blick seiner Analysen zu beschreiben. Sein Mensch ist wirklich mit Leib und Seele der Handelnde ${ }^{45}$. Durch die innere Integration und Transzendenz kann der Mensch sich durch jede Tat selbst verwirklichen. Eine Tat ist ein bewusstes Handeln, das quasi wie vor einem Gericht geschieht (mein Gewissen). Erst mit einer bewussten Handlung, wenn mein Verstand ein Urteil über die Sittlichkeit meiner aktiven Handlung fällt, vollbringe ich eine Tat. Meine Bestimmung ist es, als Person menschenwürdig zu handeln. Grundimperativ meiner Taten ist, im Anschluss an Kant, die Partizipation an der Macht der Wahrheit (Thomas von Aquin). Die Erfahrung des Selbstbesitzes und der Selbstbestimmung des Subjektes ${ }^{46}$, was dank unseres Gewissens geschieht, ermöglicht den Subjekten ihre Kreativität im Bereich der Applikation der Normen. Aufgrund der Unvollkommenheit in der Erkenntnis der Wahrheit, kann das Gewissen nicht immer das objektiv Gute erkennen. In meinem Handeln bin ich der Autor, der nicht nur eine äußerliche Wirkung vollbringt, sondern auch eine Autokreation, ich wirke auf mich selbst. Ich wirke auf mich selbst durch das Gute/Böse das ich anderen und mir selbst tue. Es liegt an der Freiheit des Menschen, trotz aller somatischen, psychischen, sozialen determinierenden Umstände sich in seinem freien Willen bewusst für das Gute zu entscheiden. Der Autor betont, ähnlich wie Kant, die Schlüsselbedeutung des freien Willens, das Pflichtbewusstsein als Sollen, das zu dem erkennenden Subjekt spricht. In meiner

${ }^{44}$ „Odpowiedzialność przed kimś, zintegrowana w głosie sumienia stawia moje własne ja w pozycji sędziego w stosunku do mojego własnego ja”. K. Wojtyła, Osoba i czyn, S. 215.

${ }^{45}$ Vgl. K. Wojtyła, in: Nissing (Hg.), Wer ist der Mensch?; Weigel, Der Papst der Freiheit, Paderborn 2011.

46 Kardinal Wojtyła ist sich der Rolle des Kant-Studiums für seine Selbstbestimmungskonzeption bewusst. Er schreibt: „To właśnie Kant przyczynił się do osobowego sensu (a pośrednio i osobowej struktury) samostanowienia”. K. Wojtyła, Osoba i czyn, S. 143, przypis 39. Siehe: J. Galarowicz, Człowiek jest osoba, S. 177. „Der Wille ist das Vermögen der Person zur Selbstbestimmung“, K. Wojtyła, in: Nissing (Hg.), Wer ist der Mensch?, München 2011, S. XLI; 16. „(...)mit dem Willensakt bestimme ich mich nicht nur auf ein Ziel hin, sondern ich bestimme auch mich selbst. Hierin besteht seine Bedeutung für die Person". Ebenda. 
Entscheidung bestimme ich mich selbst. Durch diese Fähigkeit bin ich als Subjekt dazu bestimmt die Verantwortung für meine Handlungen zu übernehmen ${ }^{47}$.

\section{Verantwortliche Liebe als Emanation personaler Würde}

Neben dem Begriff der Freiheit, um den sich die ganze Philosophie Wojtylas dreht, ist der Begriff der Teilnahme an der Liebe ${ }^{48}$ als Folge und Ziel der verantwortlichen Tat ebenso wichtig. Die Fähigkeit sich selbst zu verschenken, als eine Gabe für den anderen zu verstehen, ist verbunden mit dem Personsein - der Bestimmung des Menschen. Erst durch die Teilnahme an der Liebe verwirklicht sich der Mensch selbst, in der dialogischen Relation, ich - du, überschreite ich die Grenzen der Entfremdung, Isolation, der inneren Verschlossenheit. Erst durch diese Befähigung, die mit der Kategorie der Teilnahme (Partizipation) beschrieben wird, realisiert sich die wahre Bestimmung des Menschen. Durch die Fähigkeit zur Transzendenz ${ }^{49}$ kann der Mensch seine eigene Würde erkennen und durch die solidarische Gemeinschaft mit anderen Menschen seine freie Aufgabe als Hingabe

${ }^{47}$ „Tylko w związku z działaniem (czyli czynem) przeżywa też człowiek wartości moralne dobro i zło (...)”. K. Wojtyła, Osoba i czyn, S. 97; ,Verantwortung ist die besondere Eigentümlichkeit einer Tat, in der sich die Fähigkeit der Person zu handeln zeigt und zugleich verwirklicht". K. Wojtyła, Person und Tat, Freiburg /Br. 1981, S. 34.

${ }^{48}$ Über den Einfluss Max Schelers (Bedeutung der Gefühle) auf den Personbegriff Wojtylas schreibt K. Rymkiewicz ausführlich in seiner Dissertation, Von der Grundlegung der christlichen Ethik zur Grundlegung der philosophischen Anthropologie. Eine kritische Untersuchung zum Personbegriff bei Karol Wojtyla, Berlin 2002. „Woran lässt sich die Relevanz der schelerschen Wertauffassung für den Begriff der Person bei Wojtyla erkennen? Zunächst einmal ist festzuhalten, dass nur die Person im schelerschen System der 'Träger' von den Werten ,gut" und „böse“ ist, selbst wenn sie anders als bei Wojtyla aufgefasst wird. Darüber hinaus wird diese Relevanz auch schon durch Wojtylas scharfe Kritik an Scheler selbst zum Ausdruck gebracht, deren Folge die Feststellung ist, dass die von Scheler herausgearbeitete phänomenologische Theorie sittlicher Werte keinesfalls einwandfrei und in dieser Form auf die gleiche Diskussionsebene mit der Konzeption der Person unseres Autors zu stellen sei“. Ebenda, S. 28. „Was die erste Frage betrifft, steht es außer Zweifel, dass der Schwerpunkt der Kritik Wojtylas an Scheler sich auf die Rolle des Wollens in dessen System konzentriert (das Wollen ist eine notwendige Komponente der Konzeption der Person bei Wojtyla). Das Wollen war für Scheler nur ein Epiphänomen des Gefühlslebens“. Ebenda, S. 33.

49 „Die gegenwärtige Welt eröffnet uns einen so unermeßlichen Schauplatz von Mannigfaltigkeit, Ordnung, Zweckmäßigkeit und Schönheit, man mag diese nun in der Unendlichkeit des Raumes, oder in der unbegrenzten Theilung desselben verfolgen, daß selbst nach den Kenntnissen, welche unser schwacher Verstand davon hat erwerben können, alle Sprache über so viele und unabsehlich große Wunder ihren Nachdruck, alle Zahlen ihre Kraft zu messen und selbst unsere Gedanken alle Begrenzung vermissen, so daß sich unser Urtheil vom Ganzen in ein sprachloses, aber desto beredteres Erstaunen auflösen muß“. Kant $(\mathrm{Kr} V$ A $622 /$ B 650). 
finden ${ }^{50}$. Der isolierte, individualisierte Mensch, der sich für die transzendente Perspektive verschließt, verleugnet damit auch seine eigene transzendierende Freiheit und Verantwortlichkeit der Liebe. Die Gesellschaft droht, nach Wojtyla, damit in Alienation - Entfremdung zu verfallen, wenn sie sich ausschließlich auf Konsum und Materialismus ausrichten und zwischenmenschliche Solidarität (Liebe) schwinden lässt. „Wo die Perspektive der Hingabe seiner selbst nicht angenommen wird, wird stets die Gefahr einer egoistischen Freiheit bestehen “"51. Das Bewusstsein der eigenen Einzigartigkeit (Selbsterkenntnis des Ich) bildet die Voraussetzung dafür, auch den anderen als Ich, als Person anzuerkennen und ihm die gleichen Rechte wie mir selbst zu zugestehen. Dieses Bewusstsein der Würde des anderen Subjekts sowie meiner selbst, ermöglicht meinem Ich am Menschsein des anderen teilzunehmen. Durch diese Erfahrung komme ich zu dem Erleben des anderen als Person. Die Teilnahme ist, nach Wojtyla, hier nicht nur als Erfahrung (Erlebnis) zu verstehen, sondern auch als eine Aufforderung und Aufgabe, den anderen Menschen als ein Du (als anderes Ich) zu lieben. Solche Teilnahme ist mit dem Willen ${ }^{52}$ verbunden, das Liebesgebot „du sollst deinen Nächsten lieben wie dich selbst" zu verwirklichen. Der Begriff der Würde der Person betont die Fähigkeit des Menschen das Gute zu wählen. „Die Person“ - schreibt Wojtyla in „Liebe und Verantwortung“ - „,ist ein objektives Wesen, das als bestimmtes Subjekt mit der ganzen (äußeren) Welt eng in Verbindung steht und dank seiner Innerlichkeit und seines Innenlebens darin von Grund auf wurzelt. Dazu kommt, dass die Person so nicht nur mit der sichtbaren Welt in Verbindung steht, sondern auch mit der unsichtbaren Welt und vor allem mit

${ }^{50}$ K. Wojtyła schreibt dazu: „In der Pastoralkonstitution des Vaticanum II, Gaudium et spes, lesen wir u.a, dass der Mensch, der auf Erden die einzige von Gott um ihrer selbst willen gewollte Kreatur ist, sich selbst nur durch die aufrichtige Hingabe seiner selbst vollkommen finden kann (GS Nr.24). In diesen Worten scheint das Dokument des letzten Konzils gewissermaßen Jahrhunderte Tradition und Forschungen der christlichen Anthropologie im erhellenden Licht der göttlichen Offenbarung zusammenzufassen. Die Anthropologie des hl. Thomas von Aquin ist tief in den Wassern dieser Tradition verankert, und sie ist gleichzeitig offen für alle Errungenschaften des Menschlichen Denkens, welche die Ausarbeitungen des thomistischen Konzepts der Person weiter ausfüllen und entwickeln (...)“. K. Wojtyła, Die personale Struktur der Selbstbestimmung, in: H.G. Nissing (Hg.), Wer ist der Mensch?, S. 21.

${ }^{51}$ Johannes Paul II., Die Schwelle der Hoffnung überschreiten, Hamburg 1994, S. 227.

${ }^{52}$ Die Frage nach dem Sinn der Freiheit beantwortet Kant damit, dass sie die Heiligkeit des Willens ermögliche. N. Fischer schreibt dazu: „Heiligkeit des Willens erstrebt ein endliches Vernunftwesen, das Personen als Personen achtet. Auch wenn Kant Pflicht als die Achtung vor dem moralischen Gesetz bestimmt, so gebietet das moralische Gesetz doch grundsätzlich nichts anderes als die Achtung der Person als Person“. N. Fischer, Sein und Sinn der menschlichen Freiheit in der Philosophie Kants, S. 31. 
Gott. Dies ist ein weiteres Anzeichen dafür, dass die Person in der sichtbaren Welt etwas ganz Besonderes ist ${ }^{\text {"533 }}$.

Der Mensch wird durch die Kraft des kategorischen Imperativs zu verantwortlichen Handlungen (,Achtung geht jederzeit auf Personen, niemals auf Sachen") $)^{54}$, zur wahren Freiheit, die in Liebe mündet, bestimmt ${ }^{55}$. Für den einzelnen, sowie für die Gesellschaft stellt sich, die in seiner Würde als menschliche Person/en begründete Forderung das Gute zu wählen, um die Achtung des Menschen als Zweck seiner selbst anzuerkennen. Der Autor von „Person und Tat" betont implizit bereits in seiner Dissertation über den Glauben bei Johannes vom Kreuz, dass die Person sich offenbart in ihrer Gottesbeziehung ${ }^{56}$.

Aus dem oben Gesagten zeigt sich, dass die Teilnahme nicht nur als Aufgabe gegenüber der anderen menschlichen Person zu verstehen ist, sondern vor allem die Relation Mensch-Gott, als Personen-Beziehung betrifft ${ }^{57}$. An dieser Stelle

${ }^{53}$ K. Wojtyła, Liebe und Verantwortung. Eine ethische Studie, München 1981, S. 22.

${ }^{54} \mathrm{KpV}$ A35.

${ }^{55}$ Manche Kant-Interpreten würden hier einen Protest einlegen und nur ein einziges Gefühl hervorheben, das Sollenserlebnis vor dem moralischen Recht. N. Fischer schreibt dazu: „Zwar hebt Kant hervor, dass: alle Moralität der Handlungen in der Notwendigkeit derselben aus Pflicht und aus Achtung fürs Gesetz, nicht aus Liebe und Zuneigung zu dem, was die Handlungen hervorbringen sollen", zu setzen sei. Dennoch hat er auch die Liebe aus Gefühl oder Neigung für Gegenstände von der praktischen Liebe oder Liebe aus Pflicht unterschieden (KpV A 35)“. Fischer, Sein und Sinn der menschlichen Freiheit in der Philosophie Kants, S. 31.

${ }^{56}$ Im Jahr 1951 publizierte Karol Wojtyla einen Aufsatz zum Humanismus bei Johannes vom Kreuz, „ohne das neuscholastische Korsett und schon im Titel offener den Zugang auf den Menschen zeigend“. J. Splett, Anthropologie aus christlicher Erfahrung, in: H.G. Nissing (Hg.), S. Zekorn, Staunend vor dem Menschen, S. 33. Die drei Schlüsselerfahrungen: Erkenntnis, Liebe und die Nacht begleiten den Menschen auf seinem Weg zur Vereinigung mit Gott. Die Erkenntnis (Vernunft) wird in Zusammenhang mit dem Glauben gestellt und die Liebe mit dem Willen. Die Nacht steht auch in Bezug zum Glauben, verstanden als „abismo de fe“. K. Wojtyła, Świętego Jana od Krzyża nauka o wierze, Tow. Nauk. KUL Lublin 2000, S. 43, 96. Im Sinne Kants, wenn der Mensch die Insel seiner reinen Vernunft verlässt, muss er sich auf die Ungewissheit und den Abgrund des Glaubens einlassen, was mit Johannes vom Kreuz mit der Nacht zu beschreiben ist. Es erginge uns ähnlich, wenn wir bei Kants Vorstellung bleiben, wenn wir in eine Tiefe von $8000 \mathrm{~m}$ unter den Meeresspiegel eintauchen würden, hier hilft uns unsere natürliche Kraft der Vernunft nicht mehr, die Augen sind nur für die Inselbedingungen geschaffen. „Die ganze Definition der Nacht benutzt die Struktur des Glaubens, ihre innere Logik. Hier liegt die Begründung dafür warum gesagt wurde: der Glaube verdoppelt die Nacht. Gleichzeitig jedoch erklärt der Glaube die Nacht (...) Gott ist nicht ähnlich, ist nicht zu vergleichen mit Jemand geschaffenem, mit etwas Natürlichem, man soll also alles andere entfernen, wenn man die reine Vereinigung mit Gott sucht.” K. Wojtyła, Świętego Jana od Krzyża nauka o wierze, Tow. Nauk. KUL, Lublin 2000, S. 96.

57 Wojtyla schreibt dazu: „Die subjektive Bezugsdimension im Menschen, die es ihm ermöglicht, in eine so intime Gemeinschaft mit der Gottheit zu treten, was zweifellos neben der biologischen und psychischen Sphäre zum Menschlichen zählt". K. Wojtyła, O humanizmie św. Jana od Krzyża, in: Znak 6 (1951) [n. 27], 6-20; „Lumanesimo di San Giovanni della Croce”, 
ist zu betonen, dass der Verstand aus sich selbst und aus eigener Kraft nicht im Stande ist die Gottheit zu erreichen, wie Wojtyla in seiner Dissertation über Johannes vom Kreuz (Lehre vom Glauben) schreibt ${ }^{58}$. Dazu braucht der Mensch, laut Johannes vom Kreuz, die Kraft des Glaubens. Das Verhältnis zwischen Vernunft und Glauben erklärt der heilige Karmelit mit der Metapher, in welcher die Vernunft mit einem Kerzenständer verglichen wird, auf dem die Kerze des Glaubens steht ${ }^{59}$. Erst durch das Licht des Glaubens kann es zur höchsten liebevollen Vereinigung mit Gott kommen (Begegnung mit Gott durch Partizipation „Dios por participación“ ${ }^{60}{ }^{60}$.

Anteil an der Liebe ist für Wojtyla das Fundament aller personaler Erfahrungen des Menschen. „Nur Personen haben an der Liebe teil“"61. Die Beziehung des Menschen zu seinem Schöpfer ${ }^{62}$ ist zunächst wie zu einem Mittmenschen. In dieser personalen Kommunikation Ich-Du realisiert sich die Bestimmung des

in: Filosofia e letteratura in Karol Wojtyla, ed. A. Delogu/A. M. Morace, Vatikan 2007, S. 11. Drei Begriffe charakterisieren diese Erfahrung: Erkenntnis, Liebe und die Nacht. Vgl. J. Splett, Anthropologie aus christlicher Erfahrung, S. 33. Der Verstand ist aus sich selbst nicht im Stande die Gottheit zu erreichen, wie Wojtyla in seiner Dissertation über Johannes vom Kreuz (Lehre vom Glauben) schreibt.

58, ,...) die Vernunft, betrachtet in ihren natürlichen Eigenschaften, ist nicht fähig zur Vereinigung mit Gott. Diese Feststellung hat große Bedeutung für das Problem des Glaubens; wie wir weiter sehen werden wird diese natürliche Ungenügsamkeit der Vernunft ihre Lösung durch den Glauben und im Glauben finden.” K. Wojtyła, Swiętego Jana od Krzyża nauka o wierze, Tow. Nauk.KUL Lublin 2000, S 49. Diese Feststellung stimmt überein mit dem Kerngedanken und der Motivation Kants, die Grenze der Vernunft aufzuzeigen um Raum für den Glauben zu schaffen. Unsere Vernunft befindet sich in einer Lage, die der Philosoph aus Königsberg mit einer Insel vergleicht, auf der das Subjekt sich befindet und die weiteren (göttlichen) Perspektiven sind mit einem Ozean zu vergleichen, die die reine Kraft der Vernunft nicht zu erreichen vermag. Erst durch die praktische Vernunft, durch den Glauben kann der Mensch Gott begegnen.

${ }^{59}$ „el cual lugar, que aqui significa el entendimiento - que es el candelero donde se asienta esta candela de la fe". Zit. nach K. Wojtyła, Świętego Jana od Krzyża nauka o wierze, S. 43.

${ }^{60}$ Ebenda, S. 205.

${ }^{61} \mathrm{~K}$. Wojtyła, Osoba i czyn, S. 131.

${ }^{62}$,Würden nämlich »Gott und Ewigkeit mit ihrer furchtbaren Majestät uns unablässig vor Augen liegen (denn was wir vollkommen beweisen können, gilt in Ansehung der Gewißheit uns so viel, als wovon wir uns durch den Augenschein versichern)«, so würde die »übertretung des Gesetzes [...] freilich vermieden, das Gebotene gethan werden«; »die Gesinnung, aus welcher Handlungen geschehen sollen«, würde jedoch verfehlt, weil dann »die mehrsten gesetzmäßigen Handlungen aus Furcht, nur wenige aus Hoffnung und gar keine aus Pflicht geschehen« würden; das wiederum hätte zur Folge: »ein moralischer Werth der Handlungen aber, worauf doch allein der Werth der Person und selbst der der Welt in den Augen der höchsten Weisheit ankommt, würde gar nicht existiren« (KpVA 265).Vgl. N. Fischer, Vom Rang und vom Sinn der Gottesfrage in der Philosophie Kants, Die Gottesfrage in der Philosophie Immanuel Kants, Herder 2010, S. 4-5. 
Menschen, der das Verlangen nach wahrer Liebe in sich trägt und ohne Liebe keinen Sinn seiner Existenz finden kann ${ }^{63}$.

Die Anthropologie von Wojtyla bringt das Bewusstsein des Dramas der personalen Existenz des Menschen zum Ausdruck. Das ist die Spannung der Freiheit, die einerseits im Besitz des Menschen zu sein scheint und andererseits erst durch die wahren Taten errungen werden soll. Die dem Menschen eigene Art des Handelns, die auf seiner Würde gründet erfordert, dass er immer als ein selbstbestimmendes Wesen handelt. Der Mensch darf nicht durch den anderen instrumentalisiert werden, weil er im Sinne des Kantischen Imperativs immer ein Zweck an sich ist. Diese personalistische Norm ${ }^{64}$, die das Menschsein jedes Individuums betrifft, beschreibt die Existenz der Freiheit und der verantwortlichen Liebe als eine Teilnahme bzw. Partizipation am Guten ${ }^{65}$. Eine Erfüllung der Freiheit als Fähigkeit zur Selbstbestimmung geschieht in der Gemeinschaft (homo als ens sociale), deshalb betrifft die individuelle Handlung immer auch das Gemeinschaftswesen. Die Teilnahme am Menschsein des anderen wird damit in der Sprache Wojtylas zur „Wahl des anderen in mir selbst“. Diese Wahl ist zugleich eine Bestätigung seiner und meiner personalen Einmaligkeit/Würde. Dort wo die Autoteleologie der Person in Frage gestellt wird, tritt die Entfremdung in Erscheinung. In seiner Interpretation des Begriffes Entfremdung geht es Karol Wojtyla um die Betonung der Rolle der Gemeinschaftlichkeit. Der Mensch kann nicht isoliert, allein nur für sich selbst leben, denn seine Bestimmung ist es, als Person dem anderen $\mathrm{Du}$ zu begegnen und sich gegenseitig in verantwortlicher Liebe hinzugeben.

${ }^{63}$ Siehe: Johannes Paul II., Redemptor hominis, Rom 1979, S. 10.

${ }^{64}$ „Wenn in deinem Verhalten eine Person Objekt deines Handelns ist, vergiss niemals, dass du sie nicht bloß als Mittel, als Werkzeug behandeln darfst, sondern sei dir bewusst, dass sie ihr eigenes Ziel hat oder wenigstens haben sollte". K. Wojtyła, Liebe und Verantwortung, S. 25. Vgl. K. Wojtyła, in: Nissing (Hg.), Wer ist der Mensch?, S. XVIII.

${ }^{65}$ Augustinus sieht das oberste Gut, ähnlich wie Kant, im guten Willen. „Augustinus denkt den guten Willen wie Kant als >oberstes Gut<, über das hinaus sich jedoch noch ein >Höchstes >Vollendetes` denken läßt (vgl. KpV A 198)". N. Fischer, Der menschliche Wille als >causa primaく. Augustins Radikalisierung der Freiheitsproblematik., Vom Rang und vom Sinn der Gottesfrage in der Philosophie Kants, in: CH. Böttigheimer, N.Fischer, M. Gerwing (Hg.), Sein und Sollen des Menschen, Münster 2009, S. 8. 


\section{Fazit:}

Die gegenwärtige soziale Frage ist „radikal anthropologisch“ geworden ${ }^{66}$, schreibt Papst Benedikt XVI., auch im Sinne seines Vorgängers Johannes Paul II. Das Bewusstsein der Bedrohungen, die auch mit der biologischen Existenz des Menschen verbunden sind, führt uns zu der Annahme, dass die dominierende Reduktion der menschlichen Person auf die materiellen Bedürfnisse das Bild des Menschen zerstört und damit auch die größte ökologische Krise verursacht. Wenn der Mensch nicht mehr an seine eigene Würde und den Sinn seiner eigenen Existenz glaubt, dann wird er nicht nur für die gesamte Umwelt, sondern für sich selbst zur Bedrohung. Es ist deshalb nicht verwunderlich, dass die mangelnde Fähigkeit die „Spuren Gottes“ in der Welt wahrzunehmen, vor allem in den westlichen Kulturen, die durch Konsumismus und Positivismus dominiert werden, zu einer „Entfremdung“, „Angst“ und „Orientierungslosigkeit“ des Menschen, um die Begriffe Wojtylas zu benutzen, führt.

Unsere Aufgabe im vorliegenden Artikel war es, die Kantischen Motive im Denken Karol Wojtylas, vor allem in „Person und Tat“, aufzuzeigen. Es gibt mehrere Interpretationsversuche, die Philosophie Wojtylas dem Thomismus zuzuschreiben, es gibt auch Auffassungen, die den Einfluss Max Schelers unterstreichen und Wojtyla damit als Phänomenologen darstellen. Manche Autoren beschreiben die Philosophie des polnischen Papstes als thomistische Phänomenologie, wieder andere als phänomenologisierenden Thomismus. Es gibt kaum Untersuchungen, die die kantischen Einflüsse der Philosophie Wojtylas würdigen. Manche Autoren erwähnen Kant lediglich in einem Satz, indem sie seine Gedanken klar verdrehen und ihm im Sinne von Giovanni Sala SJ eine falsch verstandene Autonomie ${ }^{67}$ in den Mund legen, was überhaupt nichts

${ }^{66}$ Wir beobachten in den gegenwärtigen zahlreichen Krisen auch die ökologische Destruktion der Natur, die aber nicht nur die Wirklichkeit der uns umgebenden Welt bedrohen, sondern auch den Menschen, der direkt oder indirekt als Verursacher dieser Tendenzen genannt werden muss. Die Vergiftung der Umwelt und Zerstörung der zum Leben notwendigen Faktoren, ökologische Katastrophen usw. zeigen uns, dass es nicht lediglich um die Sorge und den Erhalt der allgemein verstandenen Natur geht, sondern wir müssen vor allem den Hauptverantwortlichen vor den seine eigene Freiheit begrenzenden materialistischen Ideologien schützen. Vgl. Caritas in Veritate 75, Benedikt XVI., Vatican 2009, S. 61. Vgl. G.L. Müller, Einführung zu Benedikt XVI, Spe Salvi, Augsburg 2008, S. 14-15.

${ }^{67}$ In Zeitungsartikeln insinuiert Sala sogar, Kant lege Freiheit als »absolute Autonomie« aus (Die Tagespost, 3.11.2004; Nr. 131, 10): „Im Zentrum der Ethik Kants steht die Freiheit als eine absolut aufgefasste Autonomie, die den Menschen unabhängig von Gott macht". Vgl. dagegen z.B. die wertvolle Arbeit von Max Küenburg SJ: Ethische Grundfragen in der jüngst veröffentlichten Ethikvorlesung Kants. Innsbruck 1925., S. 11. Vgl. N. Fischer, Müssen Katholiken weiterhin 
mit der Absicht des Königsberger Philosophen zu hat ${ }^{68}$. Norbert Fischer schreibt dazu, dass G. Sala Kant eine Position unterstellt, die er in Wahrheit bekämpft hat. Autonomie versteht Kant „nicht als die Selbstbestimmung einzelner Subjekte, sondern als die Selbstgesetzgebung der Vernunft, die zugleich als göttliches Gebot zu verstehen ist ${ }^{\text {“69. }}$. Unser Aufsatz versucht dem Bedarf an Studien, die den Einfluss Kants auf die Philosophie Wojtylas erklären, entgegenzukommen, um diesen eklatanten Mangel zumindest ansatzweise skizzierend zu beheben.

Kant wurde schon zu seinen Lebzeiten oft missverstanden und unterschätzt. Im Rahmen eines Artikels war es nicht beabsichtigt, auf weitere Einzelheiten der Geschichte der Ablehnung und Fehlinterpretationen einzugehen und sie breiter zu erläutern. Wir wollen lediglich daran erinnern, dass Kants Schriften auf den Index der verbotenen Bücher aufgenommen wurden ${ }^{70}$, was die Atheisten und Agnostiker verschiedener Provenienz gerne für ihre Ziele zu nutzen wussten und Kants große Leistung für ausschließlich säkulare, laizistische Weltanschauungen zu vereinnahmen suchten. Diese Situation dauert leider bis heute an, bei jenen Wissenschaftlern die sich nicht mühen die Quellen zu benutzen und so hat sich eine einseitige, verkürzte Interpretation Kants durchgesetzt und verfestigt. Alte Vorurteile scheinen sich nur sehr schwer beheben zu lassen, auch wenn bereits im Jahre 2000 ein positives Signal seitens des Vatikan (Johannes Paul II., sowie Kard. Ratzinger) zu Kant gegeben wurde. Auf die Frage Peter Seewalds antwortete der damalige Präfekt der Glaubenskongregation: „Den Ausdruck >um seiner selbst willen geschaffen hat gerade der Heilige Vater (scil. Johannes Paul II.) in den Enzykliken verschiedentlich aufgegriffen. Er hat ihn dabei von Immanuel Kant entlehnt und in einer neuen Weise weitergeführt. Kant hatte gesagt, der Mensch ist das einzige Wesen, das ein Zweck in sich selber ist, und nicht Zweck für etwas

Furcht vor Kant haben? Kants Philosophie als sancilla theologiaer, Katholische Universtät Eichstätt-Ingolstadt, Kastner 2005, S. 11.

${ }^{68}$ K. Rymkiewicz, Von der Grundlegung der christlichen Ethik zur Grundlegung der philosophischen Anthropologie. Eine kritische Untersuchung zum Personbegriff bei Karol Wojtyla, Berlin 2002, S. 19.

${ }^{69}$ N. Fischer, Müssen Katholiken weiterhin Furcht vor Kant haben? Kants Philosophie als sancilla theologiaer, S. 11.

${ }^{70}$ Papst Leo XII hat die Kritik der reinen Vernunft auf den Index librorum prohibitorum 16. Juni 1827 gesetzt und am 26. Juni 1827 offiziell publiziert. Noch 140 Jahre später, im Jahr 1963 musste A. Winter um die offizielle Erlaubnis des Vatikans die Bücher Kants lesen zu dürfen, bitten. „Der Vf. hat darum im Hinblick auf seine geplante Beschäftigung mit der Philosophie Kants die Erlaubnis erbeten und unter dem Datum vom 7.10.1963 erhalten, indizierte Bücher zu lesen und aufzubewahren“. A. Winter, Der andere Kant. Zur philosophischen Theologie Immanuel Kants, Hildesheim-Zürich-New York 2000, S. XII. 
anderes. Der Papst sagt nun: In der Tat, der Mensch ist ein Ende in sich selbst und ist nicht seinerseits noch einmal ein Zweck für etwas anderes" ${ }^{671}$.

Erst mühsam wird in den letzten Jahren, vor allem als „um Kants 200. Geburtstag Arbeiten vorgelegt wurden, die Kant als Metaphysiker, Moralisten und Religionsphilosophen begriffen" ${ }^{\text {"72, }}$, die Philosophie Kants als mit der christlichen Weltanschauung kompatibel aufgezeigt. Das Verhältnis der Katholiken zu Kant - obgleich vereinzelt und zögerlich - beginnt sich positiv zu verändern ${ }^{73}$.

Eine positive Stimme aus dem Vatikan zu Kant dokumentiert Norbert Fischer in seinem Vortag an der KU Eichstätt. Er spricht über die Antwort des jetzigen Papstes Benedikt XVI. auf sein Buch, das Kants Philosophie aus christlicher Perspektive aufzeigt: „Nachdem ich als Herausgeber von Kants Metaphysik und Religionsphilosophie dieses Buch Kardinal Ratzinger, dem damaligen Präfekten der römischen Glaubenskongregation, zugesandt hatte, erhielt ich eine Antwort, die auf ihre Weise eine unerwartete Wende im Verhältnis der Kirchenleitung zur Philosophie Kants markiert. Kardinal Ratzinger beendet sein Schreiben vom 23. März 2004 an den Herausgeber von Kants Metaphysik und Religionsphilosophie mit den Worten: „Vor allem hoffe ich, daß jüngere Philosophen und Theologen von Ihrem Buch lernen und daß ein neuer fruchtbarer Kontakt zu Immanuel Kant zustande kommt" $" 74$.

Karol Wojtylas Hauptwerk, Person und Tat, das im Jahre 1969 entstanden ist, bleibt auch heute hoch aktuell. Was ist der Mensch? Ist der Mensch frei? Was soll er tun? Diese kantischen Fragen wurden in der Einführung unserer Untersuchung gestellt. Die drei wichtigsten Ansatzpunkte der Philosophie Karol Wojtylas (Person, Freiheit, Liebe) konnten damit gut umschrieben werden. Erst durch die Perspektive, die im Glauben an den Schöpfer wurzelt und die Unantastbarkeit der Person jedes menschlichen Wesens als Fundament der Weltanschauung annimmt, können wir die wahre Ökologie - Ökologie der Person - gestalten. Der vorliegende Aufsatz erhebt selbstverständlich nicht den Anspruch das Thema in einem so begrenzten Rahmen auszuschöpfen. Im Gegenteil, durch die

${ }^{71}$ Benedikt XVI., Gott und die Welt. Glauben und Leben in unserer Zeit. Ein Gespräch mit Peter Seewald. Stuttgart u.a.2000, Neuausgabe 2005. Vgl. Fischer, Müssen Katholiken weiterhin Furcht vor Kant haben? Kants Philosophie als sancilla theologiaer, Katholische Universtät Eichstätt-Ingolstadt, Kastner 2005, S. 13.

${ }^{72}$ Ebenda, S. 8.

${ }^{73}$ Ebenda.

${ }^{74}$ Ebenda, S. 13. Vgl. Ders., Kants Rechtfertigung von Metaphysik und Religion. Die kritische Philosophie als >ancilla theologiaer?, in: N. Fischer (Hg.) Kant und der Katholizismus, Herder 2005, 19-32; A. Winter, Kann man Kants Philosophie schristlich nennen?, In: N. Fischer (Hg.), Kant und der Katholizismus, S. 33-57. 
skizzenhafte Einführung zur Philosophie des Autors von Person und Tat soll sie das Staunen über den Menschen anregen und den Leser zu weiteren Fragen und vertieften Studien einladen.

\section{Bibliography}

Aristoteles, Nikomachische Ethik, Berlin 2006.

Benedikt XVI., Caritas in Veritate, Vatican 2009.

Benedikt XVI., In Gott ist unsere Zukunft, Leipzig 2011.

Benedikt XVI., Wir müssen anders leben! Damit die Schöpfung überleben kann, Herder 2012.

Benedikt XVI., Gott und die Welt. Glauben und Leben in unserer Zeit. Ein Gespräch mit Peter Seewald. Stuttgart u.a.2000, Neuausgabe 2005.

Breuer C., Die Ökologie des Menschen, in: J. Kreiml, Neue Ansage des Glaubens, Regensburg 2012, S. 114-149.

Davies B., The Thought of Thomas Aquinas, Oxford 1992.

Dulles A., Jan Pawel II wobes tajemnicy osoby ludzkiej, „Ethos” 19 (2006) nr 4 (76), S. 39-53.

Fischer N., Müssen Katholiken weiterhin Furcht vor Kant haben? Kants Philosophie als rancilla theologiaer, Katholische Universtät Eichstätt-Ingolstadt, Kastner 2005.

Fischer N., Kants Rechtfertigung von Metaphysik und Religion. Die kritische Philosophie als sancilla theologiaer?, in: N. Fischer (Hg.) Kant und der Katholizismus, Herder 2005, S. 19-32.

Fischer N., Sein und Sinn der menschlichen Freiheit in der Philosophie Kants, in: Böttigheimer Ch., Fischer N., Gerwing (Hg.), Sein und Sollen des Menschen, Münster 2009.

Fischer N., Vom Rang und vom Sinn der Gottesfrage in der Philosophie Kants, Die Gottesfrage in der Philosophie Immanuel Kants, Herder 2010.

Fischer N., Der menschliche Wille als scausa primar. Augustins Radikalisierung der Freiheitsproblematik., Vom Rang und vom Sinn der Gottesfrage in der Philosophie Kants, in: Böttigheimer Ch., Fischer N., Gerwing M. (Hg.), Sein und Sollen des Menschen, Münster 2009.

Galarowicz J., Człowiek jest osobą, Krakow 1994.

Hołub G., Being a Person and acting as a Person, Forum Philosophicum 13 (2008).

Johannes Paul II., Redemptor hominis, Rom 1979.

Johannes Paul II., Die Schwelle der Hoffnung überschreiten, Hamburg 1994.

Kant I., Kritik der Urteilskraft, Könemann Verlag 1995.

Kant I., Kritik der reinen Vernunft, Köln 2011. 
Kant I., Kritik der praktischen Vernunft, Köln 2011.

Kupczak J., Destined for Liberty. The Human Person in the Philosophy of Karol Wojtyta/ John Paul II., Washington D.C. 2000.

Kasper W., Barmherzigkeit. Grundbegriff des Evangeliums - Schlüssel christlichen Lebens, Herder 2012.

Meadows D., Die Grenzen des Wachstums, Stuttgart 1972, The Limits to Growth. Universe Books, 1972.

Müller G.L., Einführung zu Benedikt XVI, Spe Salvi, Augsburg 2008.

Nissing H.-G. (Hg.), Einleitung und Übersetzung, in: K. Wojtyla, Wer ist der Mensch? Skizzen zur Anthropologie, München 2011, S. I-LXV.

Ong A., John Paul It's Philosophy of the Acting Person: A Personalistic Approach to Life, Edwin Mellen Press Ltd, 2008.

Rymkiewicz K., Von der Grundlegung der christlichen Ethik zur Grundlegung der philosophischen Anthropologie. Eine kritische Untersuchung zum Personbegriff bei Karol Wojtyla, Berlin 2002.

Spaemann R., Personen. Versuche über den Unterschied zwischen ,etwas “ und „jemand“. Klett-Cotta, Stuttgart 1996.

Schwartländer J., Der Mensch ist Person, Mainz 1968.

Schweidler W., Das Unantastbare: Beiträge zur Philosophie der Menschenrechte, Lit. Verlag 2001.

Schweidler W., Über Menschenwürde. Der Ursprung der Person und die Kultur des Lebens, Wiesbaden 2012.

Schweidler W., Über Menschenwürde. Der Ursprung der Person und die Kultur des Lebens, Wiesbaden 2012.

Stala J., Die Transzendenz als bestimmendes Merkmal der Person in der Anthropologie und Pädagogik Johanes Paul II, „The Person and the Challenges“ 2 (2012), S. 61-75.

Weigel G., Der Papst der Freiheit, Paderborn 2011.

Winter A., Der andere Kant. Zur philosophischen Theologie Immanuel Kants, HildesheimZürich-New York 2000.

Wojtyła K., Person und Tat, Herder 1981.

Wojtyła K., Osoba i czyn oraz inne studia antropologiczne, Towarzystwo Naukowe KUL, Lublin 2000.

Wojtyła K., A. Szostek, T. Styczeń, Der Streit um den Menschen. Personaler Anspruch des Sittlichen, Kevelaer 1979.

Wojtyła K., Person and Community: Selected Essays. New York: Peter Lang, 1993.

Wojtyła K., Liebe und Verantwortung, München 1981.

Wojtyła K., Love and Responsibility. San Francisco: Ignatius Press 1993.

Wojtyła K., Świętego Jana od Krzyża nauka o wierze, Towarzystwo Naukowe KUL, Lublin 2000. 
82

Waleszczuk Z., Globalizacja solidarności, Wrocław 2007.

Winter A., Der andere Kant. Zur philosophischen Theologie Immanuel Kants, HildesheimZürich-New York 2000, S. XII.

Winter A., Kann man Kants Philosophie >christlich`nennen?, in: N. Fischer (Hg.), Kant und der Katholizismus, Herder 2005, S. 33-57. 